\title{
Coronal accretion: the power of X-ray emission in AGN
}

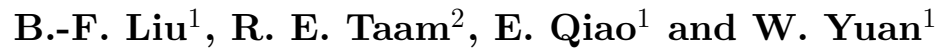 \\ ${ }^{1}$ National Astronomical Observatories, Chinese Academy of Sciences, \\ 20A Datun Road, Chaoyang District, Beijing 100012, China \\ email: bfliu@nao.cas.cn \\ ${ }^{2}$ Academia Sinica Institute of Astronomy and Astrophysics-TIARA, P.O. Box 23-141, Taipei, \\ 10617 Taiwan
}

\begin{abstract}
The optical/UV and X-ray emissions in luminous AGN are commonly believed to be produced in an accretion disk and an embedded hot corona respectively. We explore the possibility that a geometrically thick coronal gas flow, which is supplied by gravitational capture of interstellar medium or stellar wind, condenses partially to a geometrically thin cold disk and accretes via a thin disk and a corona onto the supermassive black hole. We found that for mass supply rates less than about 0.01 (expressed in Eddington units), condensation does not occur and the accretion flow takes the form of a corona/ADAF. For higher mass supply rates, corona gas condenses to the disk. As a consequence, the coronal mass flow rate decreases and the cool mass flow rate increases towards the black hole. Here the thin disk is characterized by the condensation rate of hot gas as it flows towards the black hole. With increase of mass supply rate, condensation becomes more efficient, while the mass flow rate of the coronal gas attains values of order 0.02 in the innermost regions of the disk, which can help to elucidate the production of strong X-ray with respect to the optical and ultraviolet radiation in high luminosity AGN.
\end{abstract}

Keywords. accretion, accretion disks — black hole physics - X-rays: galaxies - galaxies: active

\section{Introduction}

Accretion of gas onto a supermassive black hole is central to our understanding of active galactic nuclei (AGN). Observations of optical/UV and X-ray emissions in high luminosity AGNs point to the co-existence of hot and cool accretion flows, which have been described in terms of a hot corona lying above and below a cold standard geometrically thin disk. However, theoretical model involving the coexistence of a disk and corona as applied to luminous, radio quiet AGNs is inadequate. In particular, the inverse Compton scattering of photons from the geometrically thin disk by hot electrons in the corona quickly cools the coronal gas, if the mass supply to the accretion is dominated by a disk-like flow. In this case, the corona is too weak to emit sufficiently high X-ray radiation (Liu et al. 2012; Meyer et al. 2012). This is in contrast to the observational spectra showing that the coronal fraction in luminous AGNs can often be as high as a few tens of percent (e.g., Mushotzky et al. 1993; Elvis et al. 1994; Yuan et al. 1998). To alleviate this problem, we suggest that the physical properties of the gas fuel can play an important role in producing strong X-ray emission in AGNs. Unlike in black hole X-ray binaries where gas transferred from a companion star is cool and is mostly constrained to lie in the orbital plane, the accreting gas in AGN reflects the gravitational capture of gas from the interstellar medium or from the stellar winds emitted by evolved stars in the central regions of a galaxy (Ho 2008). In such an environment, large differences in the radiation spectrum are possible, if a thin disk exists initially. 


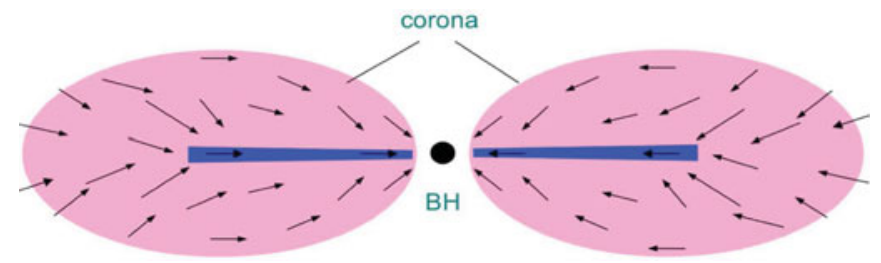

Figure 1. A schematic description of the mass flowing in the disk and corona with hot gas supply.

\section{The physical model}

We consider an accretion state in which a thin, cold disk initially surrounds a black hole. The gas supply to the accretion is from gravitational capture of the interstellar medium or the stellar winds. Such a Bondi accretion flow is hot since the accretion energy is primarily converted into internal energy due to compressional heating. As the gas flows toward the black hole, the hot gas partially condenses to the underlying disk as a consequence of disk corona interaction. Thus, the thin disk remains steady because the condensation of the hot gas continuously supplies gas to the accretion of the thin disk. On the other hand, a certain amount of hot gas remains in the corona, responding for the X-ray emission. A schematic description of the mass flowing in the disk and corona with hot gas supply are shown in Figure 1.

We study the dynamic and radiative interaction between the pre-exist disk and the hot accretion flow with mass supply from interstellar medium/stellar winds in detail. For a black hole, $M=10^{8} M_{\odot}$, viscosity parameter, $\alpha=0.3$, and magnetic field parameter (the ratio of gas pressure to total pressure), $\beta=0.8$, our calculations show that for a given mass supply rate to the corona there exists a critical distance, $R_{d}$, within which the coronal gas condenses to a cool disk. For distances greater than $R_{d}$, disk gas will evaporate to the corona. As there is only gas supply to the corona, any existing gas in a thin disk in the outer region $\left(R>R_{d}\right)$ will be evacuated by the evaporation process and hence we take $R_{d}$ as the outer boundary of the disk. The tendency for condensation resulting from efficient Compton cooling is mitigated by the thermal energy left in the corona by the condensed gas and the continuous hot gas supply preventing collapse of the corona. Thus, the accretion takes place as an ADAF with a nearly constant accretion rate in the outer region $\left(R>R_{d}\right)$, while in the inner region, a fraction of hot gas condenses to the disk as it approaches the black hole. This leads to a decreasing mass flow rate in the corona and a corresponding increase of the flow rate in the cool disk toward the black hole.

In Figure 2. we show the spatial distribution of the mass flow rate in the accretion flows for mass supply rates of $0.03,0.05,0.08$, and 0.1 . It is found that the accretion rate in the inner corona can be roughly described by a linear function, $\dot{m} \approx 0.02+3 \times 10^{-4} \frac{R}{R_{S}}$, while the mass flow rate in the innermost region of the corona is nearly independent of the mass supply rate, which converges to $\dot{m} \approx 0.02$. (in Eddington units). Such a characteristic can be understood as follows. For a higher mass supply rate, the density in the hot flow is higher, leading to the condensation of more gas to the disk by Compton cooling. Thus, the disk emission becomes stronger, which promotes more efficient Compton cooling. As a consequence, more hot gas condenses into the disk. This process stabilizes as the density of the hot flow decreases as a result of condensation and the electron temperature decreases (by efficient Compton cooling) to restrain the further increase of the Compton cooling rate. Hence, an equilibrium is established. We find that the density and mass flow rate remaining in the corona is nearly constant for the different supply rates. 


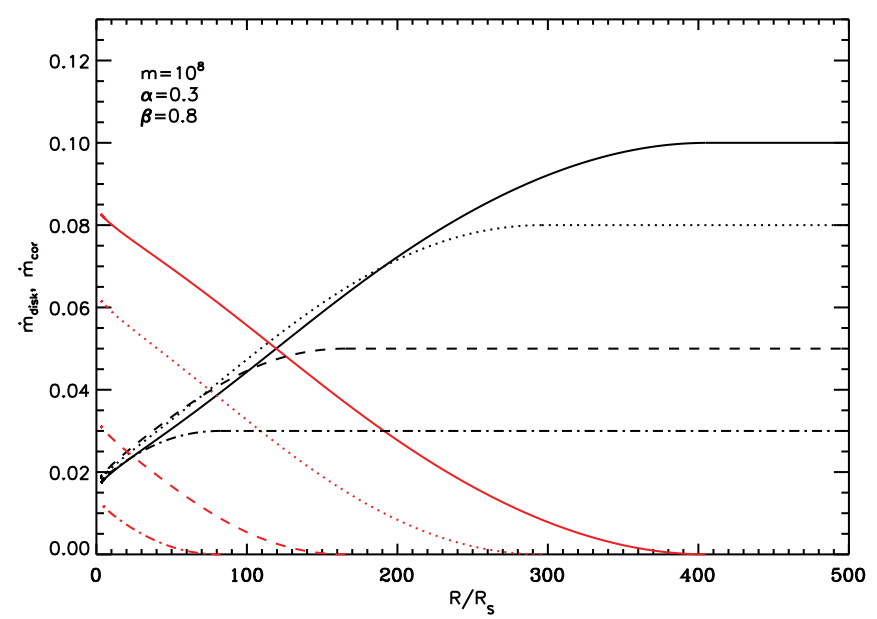

Figure 2. The spatial distribution of the mass flow rate in the disk and corona for a black hole of $10^{8} M_{\odot}$. Curves in red (black) refer to the mass flow rate in the disk (corona). From bottom to top, the dash-dotted lines refer to a hot mass supply rate of $\dot{m}=0.03$, dashed lines for $\dot{m}=0.05$, dotted lines for $\dot{m}=0.08$ and solid lines for $\dot{m}=0.1$. The mass flow rate in the corona converges to $\dot{m} \sim 0.02$ in the innermost region, indicating that a nearly constant corona coexists with a variable disk.

We point out that the above picture applies for sufficiently high accretion rates, $\dot{m}>$ 0.02 in the hot flow. For lower hot gas supply rates, $R_{d}$ is interior to the innermost stable circular orbit, implying that the coronal gas cannot condense to a disk. Instead, the interaction between the disk and corona results in the evaporation of disk gas, which evacuates the disk. Thus, at low rates of accretion, only hot accretion describes the flow, that is, the advection-dominated accretion flow.

\section{Emissions from the disk and the corona}

With the structure of the corona and disk determined, we calculate the radiation emitted from the disk and corona for $\dot{m}=0.03,0.05,0.08,0.1$. It is found that the relative importance of the corona emission depends on the mass supply rate to the corona, $\dot{m}$. With an increase of $\dot{m}$, condensation takes places at a larger distance. The ratio of corona and disk luminosity decreases with increasing mass supply rate.

The spectra are shown in Figure 3, which is composed of soft photons from the disk, Compton scattering of these soft photons, and the Compton scattering of photons produced by the synchrotron and bremsstrahlung processes in the corona. It can be seen that the strength of the emission from the hot corona relative to the disk decreases with an increase of the hot mass supply rate or bolometric luminosity. This is a consequence of the increasing condensation rate to the inner disk. The predicted value of $\alpha_{\mathrm{ox}}$, which is calculated from the luminosities at $2500 \AA$ and $2 \mathrm{keV}$, varies from 0.9 to 1.3 with the mass supply rate increasing from 0.03 to 0.1 respectively. The photon index of the hard $\mathrm{X}$-ray spectrum increases, varying from 1.8 to 2.3 in the $2-10 \mathrm{keV}$ energy band.

\section{Conclusion}

We propose that an accretion flow is supplied to a supermassive black hole by the gravitational capture of interstellar medium or stellar wind material. It is found that the accretion flow is described by a hot accretion flow in outer region while is characterized 


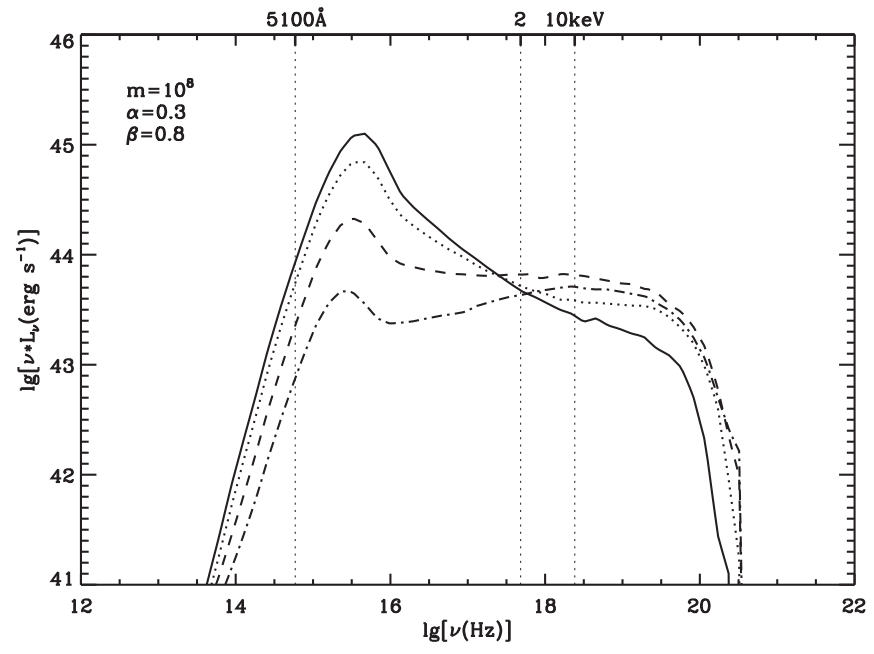

Figure 3. The spectra emitted from a disk and corona with a hot mass supply. Curves from bottom to top refer to mass supply rates of $0.03,0.05,0.08$, and 0.1 . The thin vertical lines denote the luminosity at $5100 \AA$ and $2-10 \mathrm{keV}$, showing the relative strength of the optical to hard X-ray luminosity. With an increase of the mass supply rate, the hard X-ray spectrum becomes soft (steep) and the contribution of emission from the corona decreases.

by both hot, ionized and cool gas components in the central regions of galaxies. In this picture, the hot gas partially condenses to an underlying cool disk as it flows toward the black hole, releasing accretion energy as X-ray emission and meanwhile enhancing the disk emission. Such a model can help to clarify the relative importance of the X-ray to optical/UV radiation in high luminosity AGNs, avoiding assumption that a large fraction of disk accretion energy is transferred to the corona. On the other hand, the model provides a natural interpretation of ADAF for low luminosity AGN where condensation is absent at low accretion rates.

\section{Acknowledgements}

Financial support for this work is provided by the National Natural Science Foundation of China (grants 11173029, and U1231203) and the Strategic Priority Research Program "The Emergence of Cosmological Structures" of the Chinese Academy of Sciences (Grant No. XDB09000000).

\section{References}

Elvis M. et al. 1994, ApJS, 95, 1

Ho, L. C. 2008, ARA\&A 46, 475

Liu, J. Y., Liu, B. F., Qiao, E. L., \& Mineshige, S. 2012, ApJ, 754:81

Meyer-Hofmeister, E., Liu, B. F., \& Meyer, F. 2012, A\&A, 544, A87

Mushotzky, R., Done, C., \& Pounds K. A. 1993, ARA\&A, 31, 717

Yuan, W., Brinkmann, W., Siebert, J. \& Voges, W. 1998, A\&AA,330, 108 